\title{
Results of local excision in rectal cancer. Is there good evidence?
}

$\mathrm{M}$ ost of the research in rectal cancer surgery lacks rigorous epidemiological evidence and, as a result, to use conclusions from these studies to guide decisions can be misleading. On the other hand, a recent epidemiological study has shown that in the ideal situation, randomized controlled trials can be performed to evaluate only $40 \%$ of treatment questions involving surgical procedures. Patients' preferences, abnormal conditions and lack of impartiality within the surgical community appear to be the most common reasons precluding the performance of randomized controlled trials ${ }^{1}$.

In the last few decades some authors have reported experiences with curative local resection of rectal cancer in highly selected patients. The rationale of this management is that patients with well differentiated small lesions, confined to the rectal wall, usually do not present positive lymph nodes. If we take into account that in these patients the 5- year survival rate might not differ following either abdominoperineal resection or local excision, the latter procedure would have more advantages. Patients undergoing local excision would not have colostomy, long hospital stays, nor the overall mortality/morbidity risk related to radical operations.
However, we must not jeopardize the patients'survival and, for most surgeons, a local excision procedure has to be regarded as an alternative treatment. Thus, if the patient is not fit for a major procedure or if he refuses the colostomy, the local surgical treatment is the only management that can be taken or, in other words, the need for compromise must be taken into account. Curative resection of rectal cancer implies the removal not only of the bowel segment but also of the associated limphatic drainage, which is a radical operation. This is an old but safe management, which is still followed by most rectal cancer surgeons.

Although surgeons are aware of the best trial research design for assessment of treatment effectiveness, for reasons that are not very clear they usually place great reliance on case reports and other less rigorous studies. The increasing cost of surgical treatment shows clearly the great importance of rigorous cost-effectiveness studies. In spite of potential problems that preclude the performance of ideal clinical research in the field of surgery, until randomized controlled trials can show us the comparative results between major and minor procedures in rectal cancer patients, the question regarding the efficacy of local management remains to be answered.

\section{REFERENCE}

1. Solomon MJ, McLeod RS. Should we be performing more randomized controlled trials evaluating surgical operations? Surgery 1995;118(3):459-67. 